\title{
MOMENTUM AND HEAT FLUX MEASUREMENTS IN THE EXHAUST OF VASIMR USING HELIUM PROPELLANT
}

\author{
D. Gregory Chavers", Franklin R. Chang-Díaz ${ }^{\$}$, Claude Irvine ${ }^{\dagger}$, Jared P. Squire" \\ - Research Scientist, Propulsion Research Center, MSFC/NASA, Huntsville, AL \\ ' NASA Astronaut, ASPL Director, JSC/NASA, Houston, TX \\ ${ }^{\dagger}$ Research Scientist, Propulsion Research Center, MSFC/NASA, Huntsville, AL \\ " Research Scientist, Advanced Space Propulsion Laboratory, Muniz Engineering, Houston, TX
}

\begin{abstract}
Interplanetary travel requires propulsion systems that can provide high specific impulse (Isp), while also having sufficient thrust to rapidly accelerate large payloads. One such propulsion system is the Variable Specific Impulse Magneto-plasma Rocket (VASIMR), which creates, heats, and ejects plasma to provide variable thrust and Isp, designed to optimally meet the mission requirements. The fraction of the total energy invested in creating the plasma, as compared to the plasma's total kinetic energy, is an important factor in determining the overall system efficiency. In VASIMR, this "frozen flow loss" is appreciable when at high thrust, but negligible at high Isp. The loss applies to other electric thrusters as well. If some of this energy could be recovered through recombination processes, and re-injected as neutral kinetic energy, the efficiency of VASIMR, in its low Isp / high thrust mode may be improved. An experiment is being conducted to investigate the possibility of recovering some of the energy used to create the plasma by studying the flow characteristics of the charged and neutral particles in the exhaust of the thruster. This paper will cover the measurements of momentum flux and heat flux in the exhaust of the VASIMR test facility using helium as the propellant where the heat flux is comprised of both kinetic and plasma recombination energy. The flux measurements also assist in diagnosing and verifying the plasma conditions in the existing experiment.
\end{abstract}

\section{INTRODUCTION}

As plasma is ejected from electric thrusters, the energy required to create the plasma is typically lost in the form of frozen flow losses. ${ }^{1}$ If frozen flow energy is a large fraction of the total plasma energy, the frozen flow can severely reduce the energy efficiency of the thruster. One possible method for recovering the energy currently lost in a plasma thruster is by recombining the ions and electrons via the plasma surface or volume recombination process. The momentum and heat flux quantities measured in this experiment are necessary in characterizing the flow conditions of the charged and neutral particles in the exhaust for the purpose of determining the available recombination energy in the plasma flow. The energy recovered in this experiment is realized as thermal energy on a material surface but no method is described in this paper for using the thermal energy, only to quantify the recoverable energy.

Besides quantifying the recombination energy in the flow, this paper will also discuss the flow characteristics such as ion and neutral flow velocity as verified by measurements. The flux quantities were determined using a flat plate probe placed in the flowing plasma. A brief description is given of the test facility before describing the flux tests. A force from the exhaust on the flat plate probe will be

\footnotetext{
${ }^{1}$ Humble, R. W., Henry, G. N., Larson, W. J. Space Propulsion Analysis and Design, McGraw-Hill, New York (1995) 523.
} 
determined from the momentum flux. This force will be compared to values calculated using measured plasma parameters from electrostatic probes. The force values may be used to place bounds on the expected thrust to be realized from the thruster. From the force values and known mass flow rate of the propellant, an effective specific impulse defined as

$$
I_{s p}=\frac{F}{\dot{m} g_{0}}
$$

may be determined. The specific impulse determined from this experiment is an estimate of the specific impulse realized from the plasma source (no power was given to the ion booster section of the thruster) for the combined charged and neutral particle jets. The plasma parameters will also be used to calculate a heat flow to the plate and will be compared to the measured values of heat flow. Acceleration of the ions by the ambipolar electric field will be discussed along with the collisional process of ion-neutral charge exchange which provides a mechanism for ejecting fast neutrals. The plasma-surface recombination process is the mechanism for allowing recovery of the recombination energy in this experiment and involves the neutralization of the ions at the surface of the material by the Auger neutralization process. ${ }^{2}$

\section{EXPERIMENTAL METHOD}

The experiment was conducted at the Advanced Space Propulsion Laboratory (ASPL), Johnson Space Center (JSC), Houston, Texas in the Variable Specific Impulse Magnetoplasma Rocket (VASIMR) test facility. ${ }^{3}$ The VASIMR thruster concept uses radio waves to ionize a gas by exciting helicon modes via a helicon antenna. The VASIMR team is also investigating the use of radio waves to preferentially heat the ions produced by the helicon plasma source to increase the ion exhaust velocity. Heating of the ions will provide greater propellant efficiency. ${ }^{4}$ The VASIMR test facility uses liquid nitrogen cooled, copper wound solenoids to generate an axisymmetric magnetic field. Plasma is generated near the axis of the magnet set, as shown in Figure 1, from a helicon source using rf frequencies of 13.56 or $25 \mathrm{Mhz}$ to excite the electrons sufficiently for ionization to occur. The plasma is confined radially by the magnetic field but is allowed to flow in the axial direction. The region of interest for this investigation is the exhaust region identified in Figure 1.

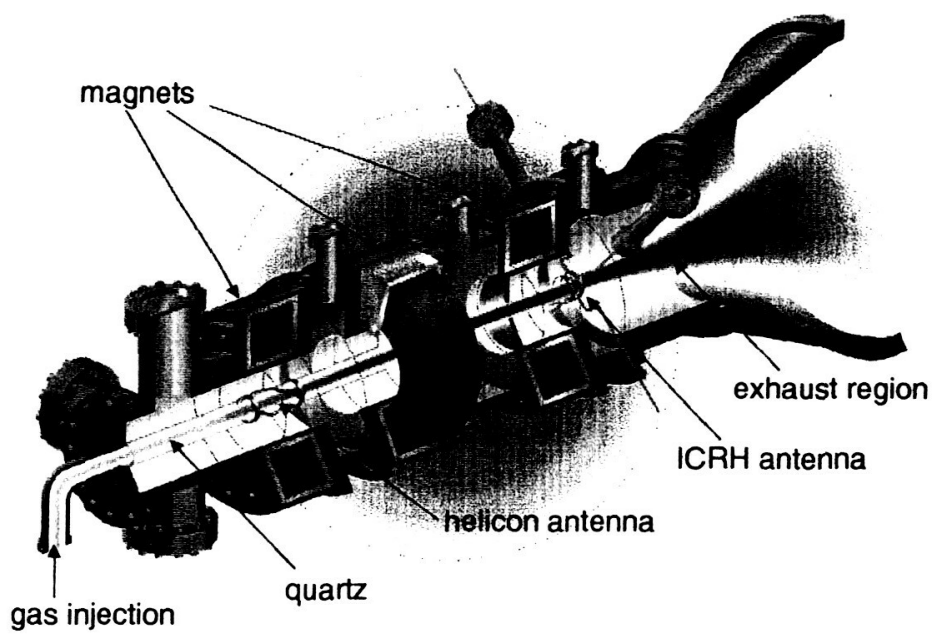

Figure 1 Schematic view of VASIMR test facility showing primary components.

\footnotetext{
${ }^{2}$ Hagstrum, H.D., Theory of Auger Ejection of Electrons from Metals by Ions. Physical Review, 1954. 96(2): p. 336-365.

${ }^{3}$ Chang-Diaz, F.R., "Research Status of The Variable Specific Impulse Magnetoplasma Rocket," Proc. $39^{\text {th }}$ Annual Meeting of the Division of Plasma Physics (Pittsburgh, PA, 1997), Bulletin of APS, 42 (1997) 2057.

${ }^{4}$ Squire, J.P., Chang-Diaz, F.R., Jacobson, V.T., McCaskill, G.E., Bengtson, R.D., Goulding, R.H., "Helicon Plasma Injector and Ion Cyclotron Acceleration Development in the VASIMR Experiment," Proceedings of the $36^{\text {th }}$ AIAA/ASME/SAE/ASEE Joint Propulsion Conference (Huntsville, Alabama 2000) AIAA 2000-3752 (2000).
} 


\section{Momentum Flux Measurement}

A momentum flux sensor was developed to measure the momentum flux of the combined neutral and charged particle jets ${ }^{5}$. This sensor consists of four uniaxial strain gages mounted on a $6 \mathrm{AL}-4 \mathrm{~V}$ titanium beam in a Wheatstone bridge configuration. An alumina rod was used to connect the titanium beam to a flat plate target. The target was then immersed in the plasma. The alumina rod serves to isolate the target electrically and thermally as well as provide a moment arm to amplify the strain in the titanium beam. The alumina rod also allows the strain gages to be located outside the plasma beam and to be shielded from radio frequency noise. Figure 2 shows a schematic of the momentum flux sensor. Through proper calibration with known forces applied to the target plate, a thrust may be inferred from the momentum flux sensor. The configuration of the test setup was not optimized to provide high thrust since the tests were primarily to determine the helicon performance as a plasma source not as a thruster by itself. However, forces of several milli-Newtons were measured on the target plate.
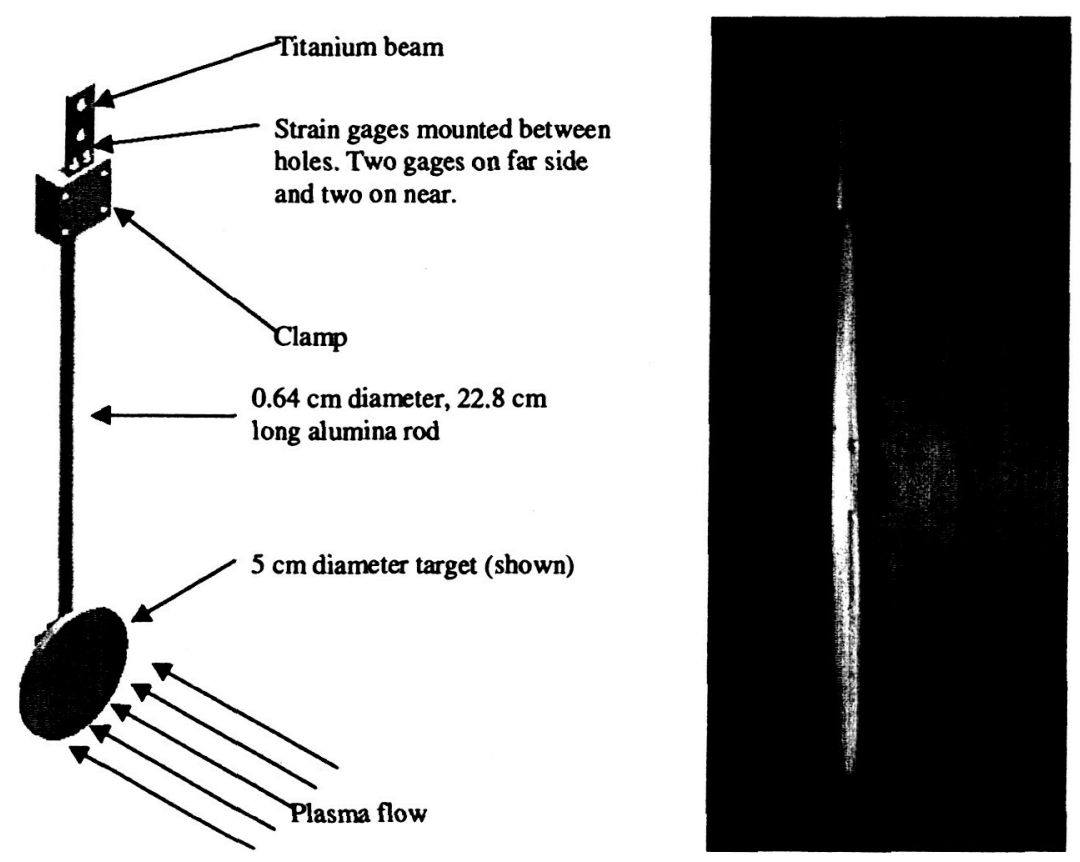

Figure 2 a)Schematic of momentum flux sensor b) $15.2 \mathrm{~cm}$ diameter target in helium plasma

The momentum flux measurement was used to verify the kinetic energy in the plasma flow. The momentum flux at the surface of the flat target may be calculated from the momentum equation given by

$$
\frac{d}{d z}\left(n_{i} m_{i} v_{i}^{2}+P_{i}+P_{e}+n_{n} m_{n} v_{n}^{2}\right)=0
$$

where ionizations have been neglected in the exhaust. The last term on the left hand side of equation (2) accounts for fast neutral particles resulting from charge exchange collisions near the plasma source. Charge exchange collisions are neglected downstream of the quartz tube exit since the plasma and neutral density decrease thereby allowing for the mean free path for collisions to increase sufficiently in this region. At the floating plate, $P_{e} \approx 0$ since most of the electrons are reflected by the sheath electric field. The sheath thickness is approximately $10 \lambda_{D}$ where the Debye length is given by

\footnotetext{
${ }^{5}$ Chavers, D.G. \& Chang-Diaz, F.R., "Momentum flux measuring instrument for neutral and charged particle flows", Rev. Sci. Instrum. 73 (10), 3500-3507
} 


$$
\lambda_{D}=\sqrt{\left(\frac{\varepsilon_{o} k_{B} T_{e}}{n e^{2}}\right)} \approx 2.5 \times 10^{-5} \text { meters }
$$

From conservation of momentum flux, the change in momentum at the plate may be equated to plasma parameters just upstream of the sheath. The change in momentum is the net force on the plate given by

$$
F=A\left(n_{e} k_{B} T_{e}+n_{i} m_{i} v_{i}^{2}+n_{n} m_{n} v_{n}^{2}\right)
$$

where the change in momentum from the sheath electric field is equal and opposite to the force caused by the surface charge density on the plate. As mentioned equation (4) provides the force on the plate based on the plasma parameters just outside the sheath with an additional force (last term on right hand side) to account for the neutral particles. The floating sheath tends to transfer power to ions from the electrons. The triple Langmuir probe was used to determine the radial density profile for the plasma terms in equation (4). More discussion of the force calculations will be given in the Results and Discussion section.

The test setup and force data collected at two axial locations are shown in Figure 3. The plasma source is near the left $(Z \sim 0)$ of each test setup and the force sensor located farther into the exhaust region measures higher force. The higher force on the most downstream force sensor is attributed to acceleration of the ions by the ambi-polar electric field and will be discussed later.

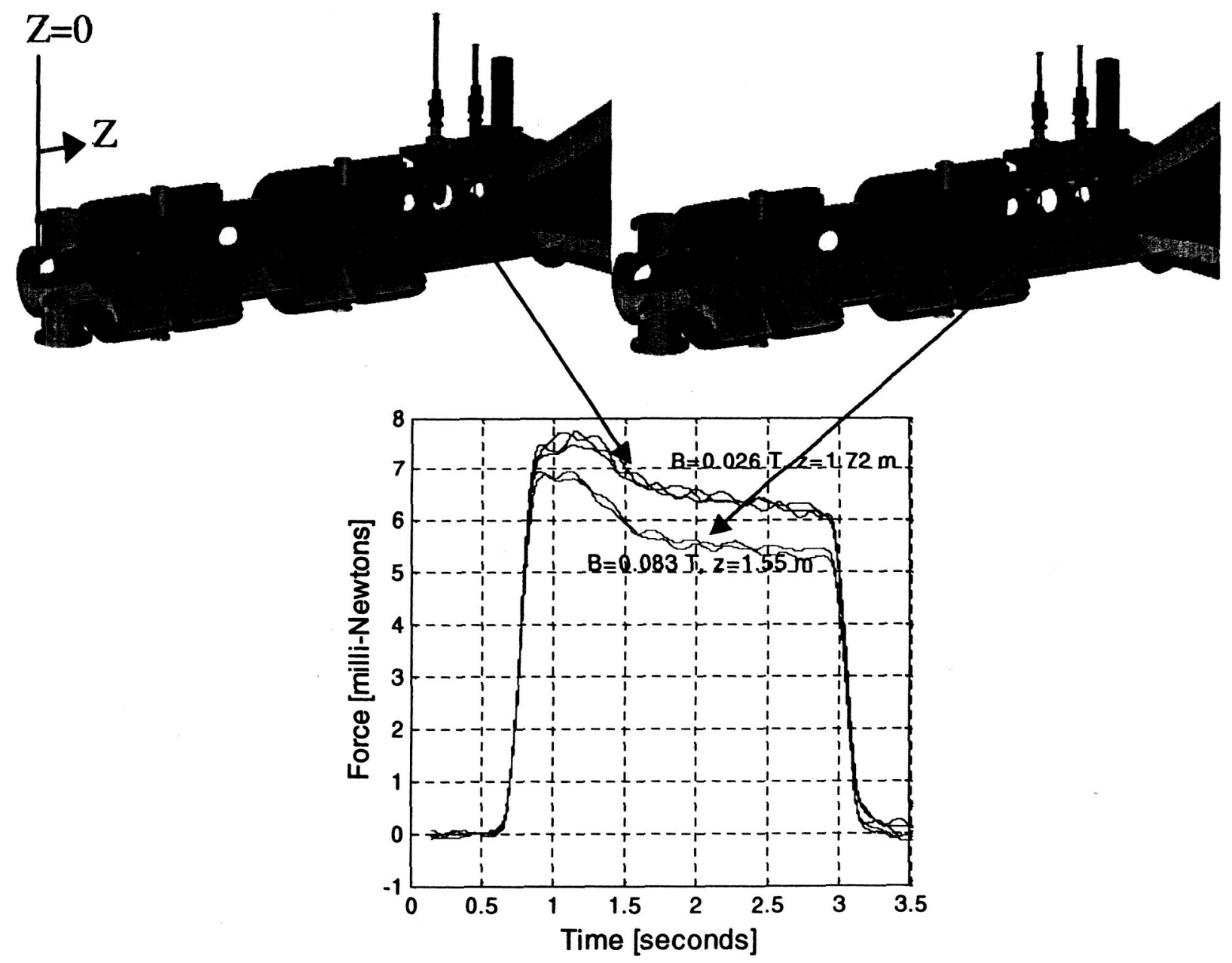

Figure 3 Test setup and force data measured at two axial locations 


\section{Heat Flux Measurement}

The heat flux to a flat plate target was determined from the change in temperature of the target measured by type $\mathrm{K}$ thermocouples connected to the rear of the target. The target was immersed in the plasma exhaust with the plasma impacting the front face of the target. The target thickness or axial dimension was 2.4 or $3.2 \mathrm{~mm}$ while target diameters of $3.18,5.1,12.7$, and $15.2 \mathrm{~cm}$ were tested. Heat flux data from the $12.7 \mathrm{~cm}$ and $15.2 \mathrm{~cm}$ diameter targets only are discussed in this paper.

The recombination energy deposited on a surface will be revealed as heat flux on the surface on which it is deposited. However, since the recombination energy cannot be measured directly, the total heat flux is measured and the contribution of the heat flux due to kinetic energy is subtracted from the total with the difference being the recombination energy. The heat flux due to kinetic energy is calculated from quantities determined by the electrostatic probes where these values are verified by the momentum flux measurement. A metallic plate was placed in the plasma flow with the surface perpendicular to the flow direction and a thermocouple was attached to the rear of the plate to measure temperature rise of the plate. Using Fourier's equation for heat conduction and an infinite slab geometry, a penetration depth, $\delta$, and penetration time, $\tau$, is given by

$$
\delta=3.64 \sqrt{\alpha t}
$$

and

$$
\tau=.0755 \frac{z^{2}}{\alpha}
$$

The penetration time of aluminum and titanium for a depth of $3.2 \mathrm{~mm}$ is determined to be 0.008 seconds and 0.093 seconds respectively. Only temperature data for the aluminum target will be discussed in this paper.

The axial thermal gradient was modeled using the one-dimensional differential equation for heat conduction in an isotropic material given by

$$
\alpha \frac{\partial^{2} q}{\partial z^{2}}=\frac{\partial q}{\partial t}
$$

The solution for equation (7) given by Carslaw and Jaeger for the temperature distribution of an infinite solid bounded by two planes. ${ }^{7}$ Based on these results the axial thermal gradient was taken to be negligible for these tests. The response of the thermocouple was different for each diameter target as was expected. The larger diameter target was oversized to allow the entire plasma beam to be intercepted. However, this larger diameter target experienced a larger radial temperature gradient in the larger target as compared to the smaller target. Even with the larger target, a uniform temperature across the target was reached within a few seconds after the plasma was turned off according to the predicted and measured values.

The smaller targets allowed the entire target to be immersed in the plasma and since the radial heat conduction was less, the target reached uniform temperature more quickly. Neglecting the axial thermal gradient and assuming axisymmetric heat flux allowed the radial thermal gradient to be modeled in one dimension. The 1-D radial heat conduction equation is given by

$$
\rho c_{p} \frac{\partial T}{\partial t}+\frac{k}{r} \frac{\partial}{\partial r}\left(r \frac{\partial T}{\partial r}\right)=S(r, t)
$$

where radiation losses are included in the source term, $S(r, t)$. The solution to equation (8) is shown graphically in Figure $4 \mathrm{a}$ for the $15.2 \mathrm{~cm}$ diameter target along with the experimental data for the

\footnotetext{
${ }^{6}$ White, F.M., Heat Transfer, Addison-Wesley, Reading, Massuchusetts (1984) 169.

${ }^{7}$ Carslaw, H.S., and Jaeger, J.C., Conduction of Heat in Solids, Oxford University Press, London (1959).
} 
thermocouple located near the radial center of the target. There are nine plasma pulses lasting 5.5 seconds with several seconds between the pulses. Targets with larger diameters than the plasma beam caused the center of the target to reach a higher temperature than the outer edges initially. The plasma beam diameter was approximately $9 \mathrm{~cm}$. The heat from the center flowed out radially until the target reached an equilibrium temperature. The model was built to verify the heat flow and time required to reach thermal equilibrium that was determined to be a few seconds. Figure $4 \mathrm{~b}$ shows the temperature data for three radially distributed thermocouples attached to the backside of the $12.7 \mathrm{~cm}$ diameter target for a single plasma shot
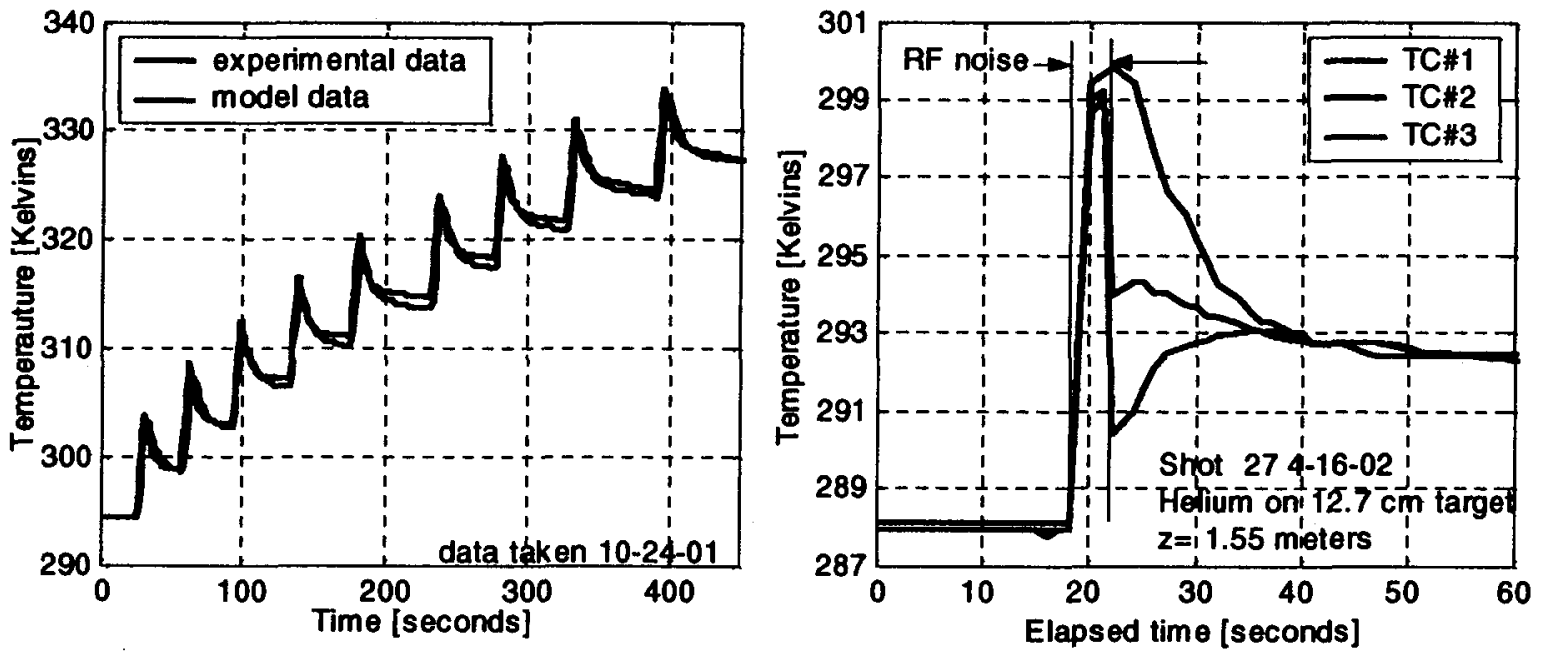

Figure 4 a)Experimental and thermal model data for $15.2 \mathrm{~cm}$ target b)thermal data for one plasma shot

\section{RESULTS AND DISCUSSION}

The heat flux and momentum flux measurements could not be taken simultaneously. An attempt to mount the thermocouple on the momentum flux target plate caused uncertainties due to forces imparted on the target plate by the thermocouple. If the force on the momentum flux sensor from the thermocouple were constant during the plasma pulse it could be subtracted as an offset. However, since the thermal expansion of the thermocouple wire as it is heated during the pulse may have induced a changing force, calibration of this changing force led to measuring the heat and momentum flux with separate instruments. The disadvantage of two separate instruments was that two separate plasma pulses were required; one for the momentum flux measurement and one for the heat flux measurement. This was acceptable as the electrostatic probes were used to verify the plasma conditions remained similar for consecutive plasma shots.

The expected force on the plate may be calculated from known quantities and compared with the measured values. A Langmuir triple probe and Mach probe are used to determine the electron temperature and density within a factor of two based on electrostatic probe theory. ${ }^{8}$ A microwave interferometer was used to calibrate the triple probe to within a factor of $10 \%$. Two plasma parameters determined from electrostatic probes were the ion rate,

$$
\Gamma_{i}=\int_{0}^{R} n_{e}(r) M(r) c_{s}(r) d r=1.3 \times 10^{19} / s,
$$

\footnotetext{
${ }^{8}$ Bering III, E.A., Brukardt, M., Chang-Diaz, F.R., Squire, J.P., Jacobson, V., Bengtson, R.D., Gibson, J.N., Glover, T.W., "Experimental Studies of the Exhaust of The VASIMR Engine," Proceedings of $40^{h}$ AlAA Aerospoce Sciences Meeting and Exhibit, Jan 14-17, 2002, Reno, NV, AIAA 2002-0345 (2002).
} 
and the average electron temperature,

$$
k_{B} T_{e}=5.3 \mathrm{eV}
$$

where $M(r)$ is the Mach number, $c_{s}(r)$ is the ion acoustic speed, and $n_{e}(r)$ is the electron density. The volume flow rate determined by the MKS flow controller was 100 standard cubic centimeters per minute (sccm). Therefore the rate of neutral particles exhausting during steady state conditions is given by

$$
\Gamma_{n}=\Gamma_{\text {otol }}-\Gamma_{i}=3.2 \times 10^{19} / \mathrm{s} \text {. }
$$

The ions will be accelerated in the exhaust by the ambipolar electric field. The ambipolar electric field may be calculated by ${ }^{9}$

$$
\vec{E}=-\frac{k_{B} T_{e}}{|e|} \frac{1}{n} \nabla n
$$

Equation (12) may be written as a change in electric potential between location (1) and location (2),

$$
\Delta \phi_{21}=\frac{k_{B} T_{e}}{|e|} \ln \left(\frac{n_{2}}{n_{1}}\right) .
$$

Since the plasma is magnetized and will follow the magnetic lines of force in the region of measurement, the diverging magnetic field will cause an axial density gradient. Using conservation of magnetic flux, the change in density is related to the change in magnetic field by

$$
n_{2}=n_{1} \frac{B_{2}}{B_{1}}
$$

The ions will then be accelerated as the magnetic field diverges. However, from continuity the acceleration of the ions will enhance the density gradient as determined by the continuity equation

$$
\frac{\partial n}{\partial t}+\nabla \cdot(n \vec{v})=0
$$

The magnetic field reaches a peak value just axially downstream of the plasma source. The ions are assumed to reach approximately the ion acoustic speed at this axial location. The ion acoustic speed is given by

$$
c_{s} \equiv \sqrt{\frac{k_{B} T_{e}}{m_{i}}} .
$$

The axial location of the peak magnetic field is just upstream of the exit of the quartz tube. The neutral pressure at this axial location is estimated to be a few millitorr providing a mean free path for ion-neutral charge exchange of only a few centimeters. The neutral pressure and density decrease just downstream of the quartz tube exit to near the background pressure in the vacuum chamber. At the mass flow rate used in this experiment, the mean free path for charge exchange is greater than 0.5 meters in the exhaust region outside the quartz tube. Therefore the fast moving neutrals are assumed to be created between the plasma source and the exit of the quartz tube. The charge exchange process involves a collision between an ion and a neutral. In this experiment the ions are moving much faster than the ambient neutrals. The fast ion acquires an electron from the slow neutral thereby neutralizing the ion. The fast moving particle is now a neutral and is not affected by the electric or magnetic fields. The fast neutral has the velocity that the ion had before the collision. The resulting ion has the velocity the neutral had before the collision and will be accelerated by the local electric field. The electrons are assumed isothermal throughout the exhaust since the mean free path for electron collisions with ions or neutrals is on the order of meters.

${ }^{9}$ Goldston, R.J. and Rutherford, P.H. Introduction to Plasma Physics, Institute of Physics Publishing, London (1995) 
Using the peak magnetic field as the reference point for determining the change in electric potential and assuming the ions are near the ion acoustic velocity at that location, the change in potential from the peak magnetic field to $z=1.55$ meters is

$$
\Delta \phi_{z=1.55}=14.4 \mathrm{~V}
$$

and the change in potential from the peak magnetic field to $\mathrm{z}=1.72$ meters is

$$
\Delta \phi_{\mathrm{z}=1.72}=21.7 \mathrm{~V} \text {. }
$$

The force on the target plate from the ions may be determined by

$$
F=\Gamma_{i} m_{i} v_{i}
$$

which gives a force at $\mathrm{z}=1.55$ meters of

$$
F_{i, z=1.55}=0.0025 \mathrm{~N}
$$

and at $\mathrm{z}=1.72$ meters of

$$
F_{i, z=1.72}=0.0030 \mathrm{~N}
$$

where the change in force is due to the ambipolar acceleration of the ions.

As mentioned, the electrons are assumed isothermal throughout the exhaust so the force from the electron pressure is given by

$$
F_{e}=A n_{e} k T_{e}=0.0011 \mathrm{~N}
$$

and is the same at both axial locations. The force from the neutrals is given by

$$
F_{n}=\Gamma_{n} m_{n} v_{n}=0.002 \mathrm{~N}
$$

where approximately $75 \%$ of the neutrals are assumed to impact the force sensor. This value is an estimate and can be accounted for by only slight radial diffusion of the neutrals. The force from the neutrals is assumed to be similar at both axial locations measured since the axial locations are only 0.17 meters apart. Therefore the total force on the plate at $\mathrm{z}=1.55$ meters is calculated to be $0.0056 \mathrm{~N}$ and at $\mathrm{z}=1.72$ meters is $0.0061 \mathrm{~N}$ while the measured values are $0.0056 \mathrm{~N}$ and $0.0063 \mathrm{~N}$ respectively. These force values compare favorably to the momentum flux sensor data which is taken to be steady state at 2 seconds into the plasma shot. A pre-puff of propellant gas at a flow rate higher than the steady state flow rate is used to initiate the plasma discharge. The plasma may reach steady state before 2 seconds but the pre-puff of propellant at the beginning of the plasma shot is observed to elevate the neutral pressure in the source region until approximately 2 seconds. The effect of the propellant pre-puff is also observed on the momentum flux sensor.

If the force shown in Figure 3 is assumed to be the thrust realized by the device, then the specific impulse for the helicon source is given by

$$
I_{s p}=\frac{F}{\dot{m} g}=\frac{0.006 \mathrm{~N}}{\left(3 \times 10^{-7} \mathrm{~kg} / \mathrm{sec}\right)\left(9.8 \mathrm{~m} / \mathrm{s}^{2}\right)}=2,000 \mathrm{sec}
$$

This value of specific impulse is comprised of the combined plasma and neutral particle jets. using

The change in temperature of the target was used to calculate the heat flux or absorbed power

$$
q(t)=m c_{p} \frac{d T}{d t}+\alpha\left(T-T_{s}\right)+\varepsilon \sigma\left(T_{p}^{4}-T_{c h}^{4}\right) A_{r}
$$


The initial temperature used in equation (25) was taken at the beginning of the plasma pulse while the final temperature was taken after the plasma pulse when the target reached thermal equilibrium (about 15 seconds). The target size and mass were $12.7 \mathrm{~cm}$ and $0.086 \mathrm{~kg}$ for the measurements analyzed in this experiment. The heat flow to the target for six consecutive plasma shots using equation (25) and measured values of temperature was determined to be $172+/-2.2$ watts.

The heat to the surface may be calculated using

$$
q_{\text {plasma }}=\Gamma_{i}\left(\left(\frac{1}{2} m_{i} v_{i}^{2}-|e| \phi_{f}\right)\left(1-R_{i E}\right)+\frac{2 k T_{e}}{1-\delta_{e}}+E_{\text {rec }}\right)
$$

where the ion temperature has been neglected and the pre-sheath acceleration has been neglected since the ion speed is greater than the ion acoustic speed before reaching the sheath. ${ }^{10} \mathrm{~A}$ pre-sheath electric field exists when plasma is flowing at speeds lower than the ion acoustic speed and will accelerate the ions to the ion acoustic speed required by the Bohm criteria at the plasma - sheath boundary ${ }^{11}$.

Using equation (26) and the parameters used to calculate the force values in equations (20), (22), and (23) the expected heat flow to the plate at $\mathrm{z}=1.55$ meters is

$$
q_{\text {net }}=q_{\text {plasma }}+q_{n}=169 \text { watts }
$$

where the neutral contribution to the heat flow, $q_{n}$, is 9.2 watts and the heat from the plasma recombination at the surface is

$$
q_{\text {rec }}=\Gamma_{i} E_{\text {rec }}=51 \text { watts }
$$

and was included in equation (27). The energy released by each ion-electron pair upon recombination for helium is $24.6 \mathrm{eV}$. As observed, the recombination energy accounts for approximately $30 \%$ of the energy in the plasma exhaust at the axial location of this experiment.

\section{SUMMARY}

The data presented in this paper shows that the momentum and energy flow determined from the experiment agrees well with calculated values providing a reasonable estimate of particle and power balance within the exhaust region of the experiment. An estimate for expected thrust, specific impulse, and available recombination energy was determined based on experiment. Several sizes of flat targets were tested at various test conditions but only a small subset was discussed in this paper. Experiments conducted to recover the recombination energy and still permit the momentum to be partially preserved by exhausting only neutral particles have been successfully performed and will be presented in a subsequent paper.

\footnotetext{
${ }^{10}$ Stangeby, P.C., The Plasma Boundary Of Magnetic Fusion Devices, Bristol and Philadelphia, Institute of Physics Publishing (2000)

${ }^{11}$ Chen, F.C. Introduction to Plasma Physics and Controlled Fusion, New York, Plenum Press (1984)
} 


\section{NOMENCLATURE}

A cross sectional area of plasma beam

$A_{r} \quad$ radiating area of target

$c_{p} \quad$ specific heat

$c$ sound speed

$E_{\text {rec }} \quad$ energy released from plasma recombination

$F$ force on momentum flux sensor

$g_{0} \quad$ acceleration due to gravity at sea level

$I_{s p} \quad$ specific impulse

$k$ thermal conductivity of material

$k_{B} \quad$ Boltzmann's constant

$m_{i} \quad$ mass of ion

$m_{n} \quad$ mass of neutral

$\dot{m} \quad$ mass flow rate

$M \quad$ Mach number of ions measured by Mach probe

$n_{e} \quad$ electron number density

$n_{i} \quad$ ion number density

$n_{n} \quad$ neutral number density

$q(x, t)$ heat flux in material as a function $x$ and $t$

$q_{\text {rex }} \quad$ power due to recombination energy

$q_{\text {nat }} \quad$ net power to surface

$P_{i} \quad$ ion pressure

$P_{e} \quad$ electron pressure

$r$ radius

$R_{i E} \quad$ energy reflection coefficient

$S(r, t)$ source term in heat conduction equation

$t$ time

$T$ temperature

$T_{c h} \quad$ temperature of chamber as viewed from

target

$T_{e} \quad$ electron temperature

$r_{i} \quad$ ion temperature

$T_{p} \quad$ temperature of target plate

$v_{i} \quad$ velocity of ion

$v_{n} \quad$ velocity of neutral

$z \quad$ axial distance

$\alpha \quad$ thermal diffusivity of material

$\delta \quad$ heat conduction penetration depth

$\phi_{f} \quad$ floating potential

$\phi \quad$ electric potential

$\pi \quad$ pi

$\tau$ thermal conduction penetration time

$\rho \quad$ mass density $\varepsilon \quad$ emissivity of target

$\varepsilon_{0} \quad$ permittivity constant

$\sigma \quad$ Stephan-Boltzmann constant

$\Gamma_{i} \quad$ ion number rate

$\Gamma_{n} \quad$ neutral number rate

$\lambda_{D} \quad$ Debye length 